\title{
Síndrome de la resección anterior baja: un alto precio del tratamiento del cáncer de recto
}

\author{
Felipe Imigo G. ${ }^{1}$ y José Tomás Larach K. ${ }^{1}$
}

1 Unidad de Coloproctología, Departamento de Cirugía Digestiva, Pontificia Universidad Católica de Chile. Santiago, Chile.

Recibido el 2 de marzo de 2018, aceptado para publicación el 8 de junio de

Correspondencia a: Dr. José Tomás Larach K. jlarach@med.puc.c

\section{Low anterior resection syndrome: a high cost of rectal cancer treatment}

In the last 30 years, oncologic outcomes of rectal cancer treatment have been significantly improved due to multimodal management and the introduction of the concept of total mesorectum excision. Although the improvements in oncological treatment and surgical techniques are undeniable, multimodal treatment results in the onset of disorders of the intestinal, sexual and urinary function in a high proportion of these patients. Up to $90 \%$ of patients undergoing a low anterior resection refer bowel disorders such as fecal incontinence, urgency, increased frequency and fragmentation of defecation. These elements are included in an entity known as the low anterior resection syndrome and specific assessment tools have been designed recently, evidencing the high incidence and prevalence of this syndrome. In the present review, we update the pathophysiology, risk factors, clinical presentation, evaluation and the alternatives of prevention and treatment of low anterior resection syndrome.

Key words: rectal cancer; anterior resection syndrome; radiotherapy; risk factors; low anterior resection syndrome; LARS.

\section{Resumen}

En los últimos 30 años el tratamiento del cáncer del recto ha mejorado significativamente gracias al manejo multidisciplinario y a la introducción del concepto de resección total del mesorrecto (RTM), logrando disminuir las tasas de recidiva local y aumentar la sobrevida. Si bien los avances oncológicos y técnicos en términos de tasas de conservación del esfínter anal son innegables, las secuelas funcionales son significativas, especialmente las relacionadas a la función intestinal, sexual y urinaria. Hasta un $90 \%$ de los pacientes sometidos a una RTM refiere secuelas intestinales cuyos síntomas se conocen como el síndrome de la resección anterior baja (SRAB). Recientemente se han diseñado sistemas de valoración específicos que han evidenciado su alta incidencia y prevalencia. En esta revisión se entrega una mirada actualizada de la fisiopatología, factores de riesgo, formas de presentación, evaluación clínica y las distintas alternativas de prevención y tratamiento del SRAB.

Palabras clave: cáncer de recto; resección anterior baja; conservación de esfínter; factores de riesgo; síndrome resección anterior baja.

\section{Introducción}

La introducción del concepto de resección total del mesorrecto (RTM) y el manejo multimodal en el cáncer de recto extraperitoneal han disminuido la recidiva local y mejorado la sobrevida en forma significativa en los últimos 30 años. En la actualidad es posible conservar el esfínter anal en más del $80 \%$ de los pacientes evitando una ostomía definitiva ${ }^{1}$. Si bien el avance en relación a los resultados oncológicos es innegable, el tratamiento multidisciplinario ha derivado en la aparición de trastornos de la función intestinal, sexual y urinaria en una alta proporción de estos pacientes ${ }^{2}$.

Durante el seguimiento a corto y largo plazo, se han observado alteraciones relevantes en la función intestinal posoperatoria de los pacientes sometidos a RTM por cáncer de recto que varían entre un 30 y $90 \%{ }^{3}$. Estas alteraciones son variadas y se extienden en un amplio espectro entre la incontinencia fecal y dificultad defecatoria, y se han agrupado en lo que se denomina como el síndrome de la resección anterior baja (SRAB). 


\section{Definición y manifestaciones clínicas}

Aunque no existe una definición formal, el SRAB corresponde a cualquier desorden de la función intestinal luego de la proctectomía. Sus componentes principales son: deposiciones fragmentadas, incontinencia a gases, urgencia defecatoria, incontinencia a deposiciones líquidas, aumento de la frecuencia de las deposiciones, constipación, tenesmo y dolor anal ${ }^{1,3}$.

Emmertsen et al., en una cohorte retrospectiva de 961 pacientes operados por cáncer de recto (con resección parcial o total del mesorrecto), encontraron que el $82,7 \%$ presentaba deposiciones fragmentadas, el $80,3 \%$ incontinencia a gases, el 70,7\% urgencia defecatoria, el $46,3 \%$ incontinencia a deposiciones líquidas, el $37 \%$ aumento en la frecuencia de las deposiciones y el 8,4\% constipación ${ }^{4}$.

\section{Epidemiología}

Los trastornos funcionales afectan a un 30 a $90 \%$ de los pacientes tratados por cáncer de recto mediante RTM, donde coexisten trastornos intestinales, sexuales y urinarios ${ }^{3,5}$. Alrededor de $45 \%$ de los pacientes presentan compromiso intestinal mayor definido como cualquier alteración que afecta la calidad de vida en forma significativa ${ }^{6}$. Además, entre un 10 a $35 \%$ de los pacientes, presentan síntomas urológicos y sexuales destacando entre los más frecuentes la incontinencia de orina, disfunción eréctil, eyaculación retrógrada y dispareunia ${ }^{2,7}$.

\section{Fisiopatología}

La génesis del SRAB es multifactorial, contribuyendo en su desarrollo fenómenos anatómicos, sensoriales y funcionales:

\section{- Disfunción del esfinter anal}

La cirugía puede producir un daño directo sobre las fibras musculares y/o denervación durante la disección pelviana. La lesión del esfínter anal interno (EAI), causaría una disminución de la presión anal de reposo e incontinencia pasiva (escurrimiento); mientras que la lesión del esfínter anal externo (EAE), desencadenaría una disminución de la presión de contracción máxima y urgencia defecatoria ${ }^{8}$. Farouk et al., en una cohorte de 39 pacientes en quienes se utilizó endograpadoras circulares para la confección de una anastomosis colorrectal, evidenciaron lesión de las fibras musculares del EAI mediante endosonografía en $18 \%$ de los pacientes en el seguimiento a 24 meses ${ }^{9}$.

\section{- Pérdida del reflejo recto-anal inhibitorio}

El reflejo recto-anal inhibitorio (RRAI) corresponde a la relajación parcial del EAI en respuesta a la dilatación rectal. Esto determina un mecanismo de "muestreo" que permite al canal anal superior discriminar entre gases y deposiciones ${ }^{10}$. Efthimiadis et al., evidenciaron una pérdida del RRAI y una disminución de la presión anal de reposo después de la resección anterior, sin embargo, estos cambios tendían a recuperarse a los 6 meses en la gran mayoría de los pacientes ${ }^{11}$. Kakodkar et al., estudiaron a 18 pacientes en el período pre y posoperatorio de la $\mathrm{RAB}$ mediante manometría anorrectal y proctometría con balón, evidenciando que la pérdida del RRAI, una menor capacitancia y una zona de alta presión del canal anal reducida, en comparación a la población normal, fueron factores predictores de malos resultados funcionales cuando persistieron hasta los 12 meses $^{12}$.

\section{- Alteraciones en la motilidad colónica}

El colon sigmoides o descendente constituye en la gran mayoría de los casos el segmento intestinal anastomosado al remanente rectal o el ano. Se han descrito trastornos de la motilidad en estos segmentos, con una respuesta posprandial aumentada asociada a un incremento de la amplitud de las ondas anterógradas de contracción y la presencia de ondas irregulares espásticas, ambas de presentación precoz, que se asocian a urgencia defecatoria, escurrimiento y múltiples evacuaciones diarias ${ }^{13-15}$.

\section{- Disminución de la capacidad y distensibilidad del remanente rectal}

La función principal del recto es actuar como un reservorio. En el posoperatorio, esta función es compartida por el remanente rectal y el colon anastomosado. Una menor capacidad y distensibilidad explicarían pérdidas por rebalse, escurrimiento, urgencia y aumento de la frecuencia defecatoria ${ }^{1}$.

\section{- Efectos de la radioterapia}

La radioterapia, mediante un mecanismo de isquemia, fibrosis y denervación, contribuye a la génesis de alteraciones intestinales y genitourina$\operatorname{rias}^{16-18}$. En el Dutch TME trial, 62\% de los pacientes sometidos a radioterapia preoperatoria y RTM presentaron incontinencia fecal en el seguimiento a 5 años, en comparación a un 39\% cuando sólo se realizó la RTM $(p<0,001)^{19}$. Emmertsen et al., evidenciaron la presencia un SRAB mayor en el 58\% de los pacientes sometidos a RA, siendo la radioterapia uno de los factores más importantes asociados a su desarrollo con un OR de $2,41^{6}$. 


\section{Factores de riesgo}

\section{- Edad}

Los menores de 65 años tienen más síntomas intestinales que los mayores, lo que se explica probablemente por la estricta selección de los pacientes añosos para la conservación esfinteriana, dado que en ellos se favorece la resección abdominoperineal en caso de dudas funcionales ${ }^{20}$. Además, se postula que los pacientes añosos tendrían menor dismotilidad colónica, con menor urgencia y frecuencia defecatoria que los pacientes más jóvenes ${ }^{21}$.

\section{- Género}

Las mujeres tienen un 35\% más riesgo de presentar SRAB mayor, lo que podría atribuirse a los antecedentes obstétricos y a una mayor prevalencia de trastornos del piso pélvico ${ }^{20,22}$.

\section{- Altura del tumor, remanente rectal y RTM}

Los tumores a menos de $5 \mathrm{~cm}$ del margen anal y la RTM, determinan una extensa disección pélvica, un menor tamaño del remanente rectal y el consecuente riesgo de denervación e isquemia local ${ }^{6,16,23}$. Un mayor tamaño del remanente rectal $(\geq 4 \mathrm{~cm})$ cuando es oncológicamente aceptable, parece otorgar mejores resultados funcionales, sin embargo, de acuerdo a un estudio de Bondeven et al., el tamaño del remanente rectal no tiene relación con la funcionalidad intestinal cuando ha habido radioterapia neoadyuvante ${ }^{24}$.

\section{- Presencia de una ileostomía de derivación}

Causa atrofia e inflamación de la mucosa colónica, conocida como "colitis por defuncionalización". El fenómeno es temporal si hay restablecimiento de la continuidad intestinal posteriormente. Por otra parte, se ha propuesto que la ileostomía derivativa genera un remodelamiento del sistema nervioso entérico con pérdida neural, desencadenando señales aberrantes que favorecerían la disfunción intestinal ${ }^{22,25}$.

\section{- Radioterapia}

Mediante isquemia, fibrosis y denervación pelviana $^{16,26}$.

\section{- Sepsis pélvica posoperatoria}

Causada generalmente por una filtración de la anastomosis que produce inflamación, fibrosis y disminución de la distensibilidad del remanente rectal y del colon anastomosado ${ }^{20,27}$.

- Otros factores asociados descritos son la quimioterapia adyuvante y el debut clínico con sintomatología obstructiva ${ }^{20,25}$.

\section{Evaluación}

El año 2012 se publicó un cuestionario específico de evaluación para el SRAB, llamado " $L A R S$ score", validado en 961 pacientes y considerando los 5 aspectos principales del SRAB: incontinencia a líquidos, a gases, frecuencia de deposiciones, fraccionamiento de la defecación y urgencia defecatoria. En base al resultado se puede clasificar a los pacientes en 3 grupos: sin SRAB (0 a 20 puntos), SRAB menor/leve (21 a 29 puntos), SRAB mayor/ grave (30 a 42 puntos $)^{4,28}$. Esta encuesta ha sido validada en múltiples países e idiomas y correlacionada con la encuesta de calidad de vida European organization for research and treatment of cancer quality of life questionnarie core module (EORTC Q1Q-C30), de manera que pacientes con SRAB menor y SRAB mayor, tienen un impacto leve o un impacto significativo en la calidad de vida, respectivamente $^{22}$. Por este motivo y por su fácil y rápida obtención, el LARS score se considera actualmente como la herramienta de elección para la evaluación funcional intestinal de los pacientes tratados por cáncer de recto (Tabla 1) ${ }^{28}$. El año 2017 López et al., realizaron la adaptación transcultural del LARS score al castellano de Chile (Tabla 2) ${ }^{29}$.

Recientemente, los grupos daneses y británicos para el estudio del SRAB, diseñaron y validaron un sistema de puntuación prequirúrgico llamado POLARS score en base al estudio de 463 pacientes ingleses y 938 pacientes daneses (disponible online, http://www.pelicancancer.org/bowel-cancerresearch/polars). Este predice el LARS score que presentará el paciente después del tratamiento en base a edad, altura tumoral, resección parcial versus total del mesorrecto, presencia o ausencia de una ostomía derivativa y el uso de radioterapia preoperatoria, permitiendo determinar grupos de riesgo y establecer planes de prevención y tratamiento ${ }^{30}$.

\section{Manejo}

El manejo del SRAB tiene dos pilares fundamentales, la prevención y el tratamiento. No existen actualmente guías para el manejo del SRAB y generalmente se utilizan terapias validadas para la incontinencia fecal y disfunción evacuatoria para su tratamiento.

\section{Prevención}

\section{Técnica quirúrgica y lesión neurológica}

Una técnica quirúrgica prolija, especialmente en tumores localmente avanzados y bajos, permitirá 
Tabla 1. LARS Score (versión original)

Q. 1: Do you ever have occasions when you cannot control your flatus (wind)?

$\square$ No, never

$\square$ Yes, less than once per week

Yes, at least once per week

7

Q. 2: Do you ever have any accidental leakage of liquid stool?

$\square$ No, never

Yes, less than once per week

Yes, at least once per week

Q. 3: How often do you open your bowels?

$\square$ More than 7 times per day (24 hours)

4-7 times per day (24 hours)

1-3 times per day (24 hours)

Less than once per day (24 hours)

Q. 4: Do you ever have to open your bowels again within one hour of the last bowel opening?

$\square$ No, never

$\square$ Yes, less than once per week

Yes, at least once per week

Q. 5: Do you ever have such a strong urge to open your bowels that you have to rush to the toilet?

$\square$ No, never

$\square$ Yes, less than once per week

Yes, at least once per week

Interpretación: 0-20 puntos = Sin LARS; 21-29 = LARS menor/leve; $30-42=$ LARS mayor/grave

minimizar el daño de los plexos hipogástricos superiores (simpáticos) durante la ligadura de la arteria mesentérica inferior cercana a la aorta, evitar la lesión de los nervios hipogástricos (simpáticos) en la entrada a la pelvis al comienzo de la disección mesorrectal, y evitar el daño de plexos hipogástricos inferiores cuyos ramos somáticos y parasimpáticos podrían dañarse durante la disección anterolateral bajo la reflexión peritoneal.

\section{Reconstrucciones intestinales}

Considerando la fisiopatología del SRAB, se han propuestos reconstrucciones intestinales distintas a la anastomosis términoterminal (ATT), que buscan aumentar la capacidad del colon anastomosado. Entre ellas, destacan la anastomosis lateroterminal (ALT), la coloplastía transversa (CT) y el reservorio colónico en J (RCJ) (Figura 1).

Hüttner et al., en un metaanálisis de 21 estudios randomizados, incluyendo 1.636 pacientes, evidenciaron que los resultados
Tabla 2. Adaptación transcultural al español "chileno" del LARS Score

1. ¿Existen momentos en los que no puede controlar los gases por el ano?

$\square$ No, nunca

Sí, menos de una vez por semana 4

Sí, más una vez por semana

2. ¿Ha presentado alguna vez pérdida accidental de deposiciones líquidas?

$\square$ No, nunca

Sí, menos de una vez por semana

Sí, más una vez por semana

3. ¿Con qué frecuencia va al baño a defecar?

$\square$ Más de 7 veces por día (24 h)

4 a 7 veces por día $(24 \mathrm{~h})$

1 a 3 veces por día (24 h)

Menos de una vez por día (24 h)

4. ¿Ha tenido que volver al baño a defecar antes de transcurrida una hora de la última deposición?

$\square$ No, nunca

Sí, menos de una vez por semana

Sí, más una vez por semana

5. ¿Alguna vez ha sentido una necesidad tan urgente de defecar que debe apurarse para llegar al baño?

$\square$ No, nunca

Sí, menos de una vez por semana

Sí, más una vez por semana

Interpretación: 0-20 puntos $=$ Sin LARS; $21-29=$ LARS menor/leve; 30-42 = LARS mayor/grave.

funcionales luego de la $\mathrm{RAB}$, son mejores para cualquier tipo de reconstrucción (ALT, RCJ o CT) distinta de la ATT, al menos hasta los 12 meses posoperatorios, sin aumentar la morbimortalidad del procedimiento. Cabe destacar que los autores no logran comparar los resultados de las distintas técnicas a largo plazo por falta de evidencia ${ }^{31}$.

\section{Tratamiento}

\section{Medidas dietético-farmacológicas}

Asociado a una alimentación acorde al síntoma predominante, se considera el uso de fármacos laxantes o loperamida de acuerdo al caso. Los suplementos de fibras, supositorios de glicerina y el uso de polietilenglicol facilitarían la evacuación. Una opción efectiva en aquellos pacientes con dificultad evacuatoria es el uso de enemas agua (20 a $50 \mathrm{cc}$ ), que favorecerían 


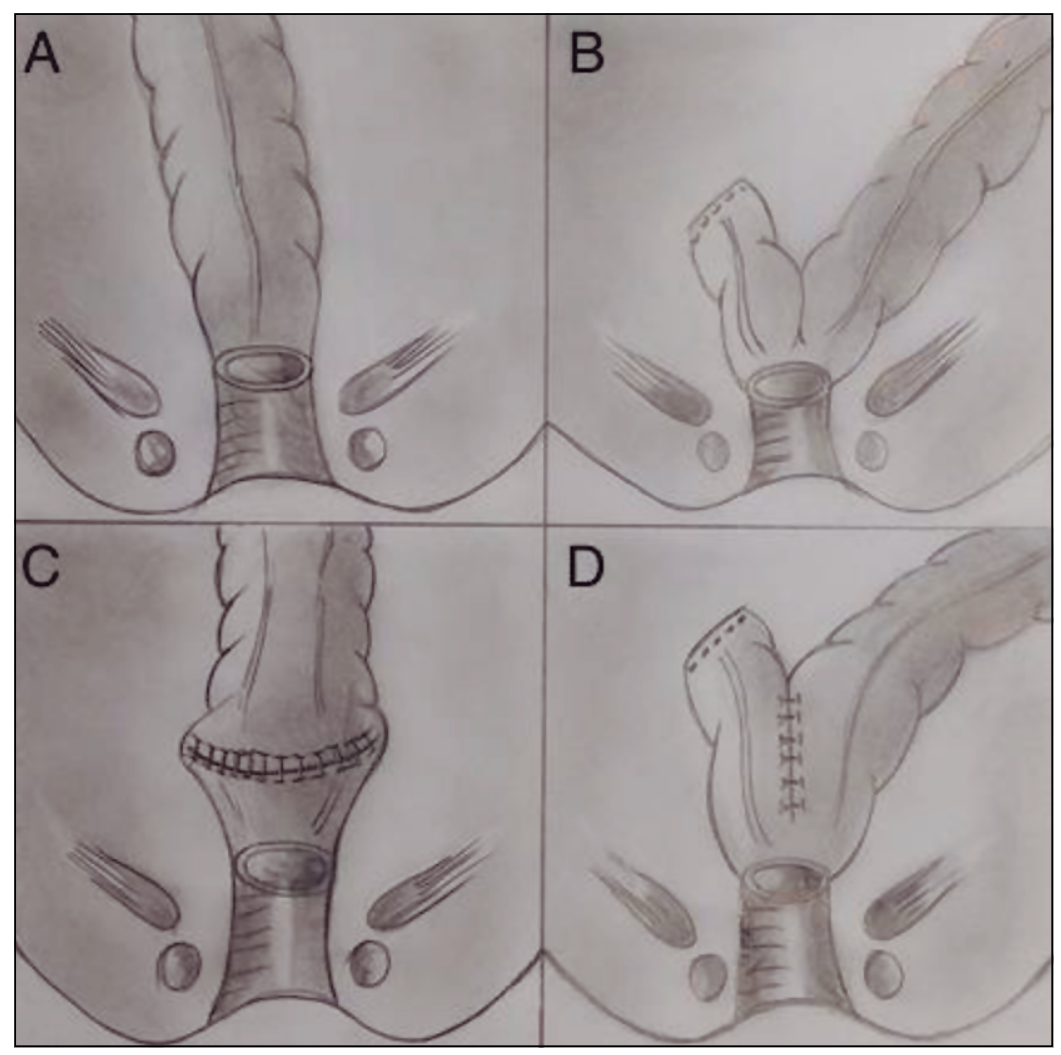

Figura 1. Reconstrucciones intestinales luego de la resección anterior baja. A. Anastomosis término-terminal; B. Anastomosis latero-terminal; C. Coloplastía transversa; D. Reservorio colónico en J. permite mejorar la sensibilidad y función del remanente ${ }^{35}$.

La primera respuesta clínica es la disminución de la urgencia fecal y, posteriormente, la frecuencia de las deposiciones diarias ${ }^{36}$. La RPP mejora en forma significativa los resultados funcionales, independientemente de la escala de evaluación utilizada ${ }^{37,38}$.

\section{Irrigación colónica transanal}

Consiste en infundir 500 a $1.500 \mathrm{ml}$ de suero fisiológico a través del ano mediante una sonda o dispositivo especialmente diseñado para este propósito (Ej. Peristeen, Colosplast), permitiendo vaciar el intestino grueso distal. La técnica de uso es simple, pero requiere periodicidad (cada 24 a $48 \mathrm{~h}$ ) y de un espacio físico cómodo para llevarla a cabo. Las complicaciones son leves, siendo las más frecuentes náuseas y dolor abdominal causadas por la distensión del intestino. La perforación intestinal es anecdótica ${ }^{36,39}$. Se ha demostrado que $50 \%$ de los pacientes logran continencia completa y disminuyen en forma significativa el número de deposiciones diarias y nocturnas en algunos estudios ${ }^{40}$.

\section{Estimulación del nervio tibial posterior}

Procedimiento no invasivo y con resultados probados en la incontinencia urinaria y fecal. Altomare et al., presentaron un estudio piloto donde demuestran una mejoría de la sintomatología intestinal y urinaria en pacientes tratados por cáncer de recto ${ }^{41}$.

\section{Estimulación de las raíces sacras}

Estudiado principalmente como tratamiento miento causado por las deposiciones remanentes ${ }^{32}$. Se ha considerado el uso de antibióticos en algunos pacientes con síntomas sugerentes de síndrome de intestino irritable, específicamente la neomicina o rifaximina, sin embargo, la evidencia es poco consistente $^{32,33}$.

Itagaki et al., reportaron en 25 pacientes masculinos sometidos a RTM una disminución de la frecuencia defecatoria, de 10,8 a 5,7 episodios al día $(\mathrm{p}<0,01)$ con la administración de ramosetron (antagonista de la serotonina) luego de 1 mes de terapia, sugiriendo una buena respuesta clínica en pacientes con aumento de la frecuencia como síntoma predominante ${ }^{34}$.

\section{Rehabilitación kinésica pelviperineal (RPP)}

Tiene tres pilares fundamentales: el entrenamiento de la musculatura pélvica, para mejorar el tono, coordinación y tiempo de la contracción muscular; biofeedback, mediante la cual el paciente permite mejorar la sensación sobre su musculatura pélvica; y el entrenamiento con balón rectal, que para la incontinencia fecal. El sistema consiste de unos electrodos que se ubican en la proximidad de las raíces nerviosas de S3 y S4, que envían pulsos eléctricos coordinados por un transductor ubicado en un bolsillo subfascial a nivel del hueso iliaco. Así, mejora la sintomatología a través de una combinación de neuromodulación local, como modificación de la actividad nerviosa central mediante estimulación aferente, aumentando las ondas peristálticas retrógradas, la continencia y frecuencia de deposiciones ${ }^{42}$.

La estimulación sacra produce mejoría subjetiva y objetiva de los síntomas con una disminución de la defecación nocturna, fragmentación, urgencia defecatoria y del escurrimiento ${ }^{43}$. Es una técnica invasiva que requiere de un operador con experiencia para su instalación. La tasa de complicaciones es de alrededor de $30 \%$ y requiere reintervenciones en cerca de $20 \%$ de los pacientes con retiro del dispositivo por infección (desde infecciones de la herida operatoria hasta meningitis) en el $4 \%$ de los $\operatorname{casos}^{44}$. 
Por el momento es considerado un tratamiento de segunda línea y como alternativa a la ostomía definitiva ${ }^{45}$.

\section{Ostomía definitiva}

Se considera cuando las medidas menos invasivas bien implementadas han fallado. Celerier et al., en un seguimiento a 10 años, mostraron que un $22 \%$ de los pacientes a quienes se les realizó una cirugía con preservación de esfínter por cáncer de recto, requirieron una ostomía permanente. En $8 \%$ se requirió una ostomía definitiva como consecuencia de trastornos de la función intestinal ${ }^{46}$.

\section{Nuevas perspectivas}

\section{- Radioterapia más selectiva}

Una correcta interpretación de la resonancia nuclear magnética (RNM) por radiólogos especialistas determina la factibilidad de llevar a cabo la cirugía sin la necesidad de radioterapia cuando no hay amenaza del margen circunferencial (cT3a-b), logrando resultados oncológicos satisfactorios a largo plazo en términos de control local, sin embargo, esta conducta no es considerada un estándar de tratamiento en este momento ${ }^{47}$. Actualmente, está en curso un protocolo multicéntrico, randomizado, que pretende evaluar la posibilidad de prescindir de la radioterapia en pacientes con cáncer de recto en etapas II y III sin amenaza del margen circunferencial, determinado por la RNM preoperatoria ${ }^{48}$.

\section{- Nuevos abordajes quirúrgicos}

Algunos trabajos han sugerido mejoras en los resultados funcionales de los pacientes tratados por cáncer de recto cuando la cirugía se ha realizado mediante el uso de robot o plataformas de acceso transanal, sin embargo, por el momento no hay evidencia categórica que avale su superioridad con respecto a la cirugía abierta o laparoscópica tradicional ${ }^{49,50}$

\section{- Monitoreo neurológico intraoperatorio}

Se ha estudiado como una alternativa para la prevención de lesiones neurológicas durante la cirugía del cáncer de recto, con resultados promisorios ${ }^{51}$. Se realiza mediante electroestimulación intraoperatoria de los nervios autónomos y observación de un patrón de electromiografía del esfínter anal interno y manometría vesical. Actualmente, existe un estudio randomizado en curso que pretende evaluar el impacto de esta estrategia en los resultados funcionales intestinales y genitourinarios de los pacientes operados por cáncer de recto ${ }^{52}$.

\section{- Preservación del órgano}

La preservación del órgano supone mejores resultados funcionales a equivalencia de resultados oncológicos en ciertos casos:

- Resección local: Se ha propuesto la resección local del cáncer de recto (submucosa o de pared total) como tratamiento oncológicamente equivalente a la RTM cuando se cumplen los siguientes criterios: adenocarcinoma bien o moderadamente diferenciado, menor de $3 \mathrm{~cm}$, que compromete menos de un tercio de la circunferencia del recto, T1 con infiltración de menos de $1.000 \mu \mathrm{m}$ en la submucosa ( $\mathrm{sm} 1)$ y sin permeaciones linfáticas o vasculares. Aunque no está claro por el momento el rol de la resección local en pacientes tratados mediante radioterapia neoadyuvante o el rol de la radioterapia adyuvante en los casos de resección local cuando hay factores histológicos de mal pronóstico, ésta es un área en pleno desarrollo ${ }^{53}$.

- Watch \& Wait: La respuesta patológica completa a la radioquimioterapia neoadyuvante ocurre en aproximadamente $15 \%$ de los pacientes tratados por cáncer de recto ${ }^{54}$. Por ahora, no hay mecanismos, fuera del análisis de la pieza operatoria, que permitan discriminar totalmente aquellos pacientes en los que hay una respuesta patológica completa de aquellos en que todavía hay viabilidad tumoral, a pesar de que no se identifique tumor clínicamente (tacto rectal, endoscopía e imágenes). La respuesta clínica completa no predice una respuesta patológica completa en todos los casos y aproximadamente un tercio de los pacientes tienen recidivas en el seguimiento, siendo rescatables aproximadamente el $83 \%$ de los $\operatorname{casos}^{55}$. Por ahora, la estrategia de Watch \& Wait no se considera un estándar de tratamiento, pero es un área donde veremos avances importantes en el futuro.

\section{Conclusión}

El SRAB es una secuela funcional frecuente y potencialmente invalidante en los pacientes operados por cáncer de recto.

Además, de las expectativas oncológicas del tratamiento, es fundamental incluir en la conversación preoperatoria con el paciente, las eventuales complicaciones funcionales intestinales, urinarias y sexuales que podría presentar luego de la proctectomía.

Considerando la alta incidencia de esta entidad se deben realizar esfuerzos para identificar aquellos pacientes de mayor riesgo aplicando sistemas de 
evaluación validados, permitiendo de esta forma obtener una valoración objetiva y establecer estrategias de prevención y tratamiento precoz.

En el futuro veremos cómo nuevas estrategias multidisciplinarias para la preservación del órgano y el avance de la tecnología aplicada a la cirugía (cirugía mediante plataformas transanales, robótica y monitoreo neurológico), podrían tener un impacto importante en los resultados funcionales de los pacientes tratados por cáncer de recto.

\section{Responsabilidades éticas}

Protección de personas y animales. Los autores declaran que para esta investigación no se han realizado experimentos en seres humanos ni en animales.

Confidencialidad de los datos. Los autores declaran que en este artículo no aparecen datos de pacientes.

Conflictos de interés: no hay.

\section{Referencias}

1. Bryant CLC, Lunniss PJ, Knowles CH, Thaha MA, Chan CLH. Anterior resection syndrome. Lancet Oncol 2012;13(9):e4038. http://dx.doi.org/10.1016/S14702045(12)70236-X.

2. Adam JP, Denost Q, Capdepont M, van Geluwe B, Rullier E. Prospective and Longitudinal Study of Urogenital Dysfunction After Proctectomy for Rectal Cancer. Dis Colon Rectum 2016;59:82230. http://www.ncbi.nlm.nih.gov/ pubmed/27505110.

3. Ridolfi T, Berger N, Ludwig K. Low Anterior Resection Syndrome: Current Management and Future Directions. Clin Colon Rectal Surg 2016;29:23945. http://www.thieme-connect.de/DOI/ DOI? $10.1055 / \mathrm{s}-0036-1584500$.

4. Emmertsen KJ, Laurberg S. Low Anterior Resection Syndrome Score. Ann Surg. 2012;255:922-8.

5. Juul T, Ahlberg M, Biondo S, Espin E, Jimenez LM, Matzel KE, et al. Low anterior resection syndrome and quality of life: an international multicenter study. Dis Colon Rectum 2014;57:585-91. http://eutils.ncbi.nlm.nih.gov/entrez/ eutils/elink.fcgi?dbfrom=pubmed\&id=2 4819098\& retmode $=$ ref $\& \mathrm{cmd}=$ prlinks $\%$ 5Cnpapers3://publication/doi/10.1097/ DCR.0000000000000116.

6. Emmertsen KJ, Laurberg S. Impact of bowel dysfunction on quality of life after sphincter-preserving resection for rectal cancer. Br J Surg. 2013;100:1377-87.

7. Ozgen Z, Ozden S, Atasoy BM, Ozyurt H, Gencosmanoglu R, Imeryuz N. Long-term effects of neoadjuvant chemoradiotherapy followed by sphincter-preserving resection on anal sphincter function in relation to quality of life among locally advanced rectal cancer patients: a cross-sectional analysis. Radiat Oncol 2015;10:168. http://www.pubmedcentral.nih.gov/ articlerender.fcgi?artid $=4554367 \&$ tool $=p$ mcentrez\&rendertype $=$ abstract.

8. Horgan PG, O'Connell PR, Shinkwin CA, Kirwan WO. Effect of anterior resection on anal sphincter function. Br J Surg 1989;76:783-6. http://www.ncbi.nlm.nih. gov/pubmed/2765827.

9. Farouk R, Duthie GS, Lee PW, Monson JR. Endosonographic evidence of injury to the internal anal sphincter after low anterior resection: long-term follow-up. Dis Colon Rectum 1998;41:888-91. http:// www.ncbi.nlm.nih.gov/pubmed/9678375.

10. Kaur G, Gardiner A, Duthie GS. Rectoanal reflex parameters in incontinence and constipation. Dis Colon Rectum 2002;45:928-33. http://www.ncbi. nlm.nih.gov/pubmed/12130882.

11. Efthimiadis C, Basdanis G, Zatagias A, Tzeveleki I, Kosmidis C, Karamanlis E, et al. Manometric and clinical evaluation of patients after low anterior resection for rectal cancer. Tech Coloproctol. 2004;8(SUPPL. 1):205-7.

12. Kakodkar R, Gupta S, Nundy S. Low anterior resection with total mesorectal excision for rectal cancer: functional assessment and factors affecting outcome. Color Dis 2006;8:6506. http://doi.wiley.com/10.1111/j.14631318.2006.00992.x.

13. Lee WY, Takahashi T, Pappas T, Mantyh CR, Ludwig KA. Surgical autonomic denervation results in altered colonic motility: an explanation for low anterior resection syndrome. Surgery 2008;143:778-83. http://www.ncbi.nlm. nih.gov/pubmed/18549894.
14. Iizuka I, Koda K, Seike K, Shimizu K, Takami Y, Fukuda H, et al. Defecatory malfunction caused by motility disorder of the neorectum after anterior resection for rectal cancer. Am J Surg 2004;188:17680. http://www.ncbi.nlm.nih.gov/ pubmed/15249246.

15. Emmertsen KJ, Bregendahl S, Fassov J, Krogh K, Laurberg S. A hyperactive postprandial response in the neorectum - the clue to low anterior resection syndrome after total mesorectal excision surgery? Color Dis 2013;15:e599606. http://www.ncbi.nlm.nih.gov/ pubmed/23869468.

16. Lange MM, den Dulk M, Bossema ER, Maas CP, Peeters KCMJ, Rutten HJ, et al. Risk factors for faecal incontinence after rectal cancer treatment. Br J Surg. 2007;94:1278-84. http://www.ncbi.nlm. nih.gov/pubmed/17579345.

17. Bernard S, Ouellet MP, Moffet H, Roy JS, Dumoulin C. Effects of radiation therapy on the structure and function of the pelvic floor muscles of patients with cancer in the pelvic area: a systematic review. J Cancer Surviv 2016;10:35162. http://www.ncbi.nlm.nih.gov/ pubmed/26314412.

18. Andreyev HJN, Benton BE, Lalji A, Norton C, Mohammed K, Gage H, et al. Algorithm-based management of patients with gastrointestinal symptoms in patients after pelvic radiation treatment (ORBIT): A randomised controlled trial. Lancet 2013;382:2084-92. http://dx.doi. org/10.1016/S0140-6736(13)61648-7.

19. Peeters KCMJ, van de Velde CJH, Leer JWH, Martijn H, Junggeburt JMC, Kranenbarg EK, et al. Late side effects of short-course preoperative radiotherapy combined with total mesorectal excision 
for rectal cancer: Increased bowel dysfunction in irradiated patients - A Dutch Colorectal Cancer Group Study. J Clin Oncol. 2005;23:6199-206.

20. Bregendahl S, Emmertsen KJ, Lous J, Laurberg S. Bowel dysfunction after low anterior resection with and without neoadjuvant therapy for rectal cancer: A population-based cross-sectional study. Colorectal Dis. 2013;15:1130-9.

21. Yu S, SS. R. Anorectal physiology and pathophysiology in the elderly. Clin Geriatr Med. 2014;30:95-106. http:// dx.doi.org/10.1016/j.cger.2013.10.003

22. Battersby NJ, Juul T, Christensen P, Janjua AZ, Branagan G, Emmertsen $\mathrm{KJ}$, et al. Predicting the Risk of BowelRelated Quality-of-Life Impairment After Restorative Resection for Rectal Cancer: A Multicenter Cross-Sectional Study. Dis Colon Rectum 2016;59:270-80.

23. Tomita R, Igarashi S, Fujisaki S. Studies on anal canal sensitivity in patients with or without soiling after low anterior resection for lower rectal cancer. Hepatogastroenterology. 2008;55:13114. http://www.ncbi.nlm.nih.gov/ pubmed/18795679.

24. Bondeven P, Emmertsen KJ, Laurberg S, Pedersen BG. Neoadjuvant therapy abolishes the functional benefits of a larger rectal remnant, as measured by magnetic resonance imaging after restorative rectal cancer surgery. Eur J Surg Oncol. 2015;41:1493-9. http://dx.doi. org/10.1016/j.ejso.2015.07.003.

25. Wells CI, Vather R, Chu MJJ, Robertson JP, Bissett IP. Anterior Resection Syndrome-A Risk Factor Analysis. J Gastrointest Surg. 2014;19:350-9.

26. Hughes DL, Cornish J, Morris C, LARRIS Trial Management Group. Functional outcome following rectal surgerypredisposing factors for low anterior resection syndrome. Int J Colorectal Dis. 2017. http://www.ncbi.nlm.nih.gov/ pubmed/28130593.

27. Ziv Y, Zbar A, Bar-Shavit Y, Igov I. Low anterior resection syndrome (LARS): Cause and effect and reconstructive considerations. Tech Coloproctol. 2013;17:151-62.

28. Juul T, Ahlberg M, Biondo S, Emmertsen KJ, Espin E, Jiménez LM, et al. International validation of the low anterior resection syndrome score. Ann Surg.
2014;259:728-34. http://eutils.ncbi.nlm. nih.gov/entrez/eutils/elink.fcgi?dbfrom= pubmed $\&$ id $=23598379 \&$ retmode $=$ ref $\&$ cmd=prlinks\%5Cnpapers3://publication/ doi/10.1097/SLA.0b013e31828fac0b.

29. López S, Carrillo K, Sanguineti A, Azolas R, Díaz M, Bocic G, et al. Adaptación transcultural del cuestionario acerca de la función intestinal (LARS Score) para su aplicación en pacientes operados de cáncer de recto medio y bajo. Rev Chil Cir. 2017;69:44-8.

30. Battersby NJ, Bouliotis G, Emmertsen KJ, Juul T, Glynne-Jones R, Branagan G, et al. Development and external validation of a nomogram and online tool to predict bowel dysfunction following restorative rectal cancer resection: the POLARS score. Gut 2017;gutjnl-2016-312695. http:/gut.bmj.com/lookup/doi/10.1136/ gutjnl-2016-312695.

31. Hüttner FJ, Tenckhoff S, Jensen K, Uhlmann L, Kulu Y, Büchler MW, et al. Meta-analysis of reconstruction techniques after low anterior resection for rectal cancer. Br J Surg. 2015;102:735-45.

32. Emmertsen KJ, Laurberg S. Identifying and Treating Patients With Pelvic Organ Dysfunction After Treatment for Pelvic Cancer. Dis Colon Rectum 2016;59:83-5. http://www.ncbi.nlm. nih.gov/pubmed/26651117\%5Cnhttp:// content.wkhealth.com/linkback/op enurl?sid=WKPTLP:landingpage\& an $=00003453-201601000-00013$.

33. Martellucci J. Low Anterior Resection Syndrome a treatment alorith. Dis Colon Rectum 2016;59:79-82.

34. Itagaki R, Koda K, Yamazaki M, Shuto K, Kosugi C, Hirano A, et al. Serotonin (5-HT3) receptor antagonists for the reduction of symptoms of low anterior resection syndrome. Clin Exp Gastroenterol. 2014;7:47-52.

35. Scott KM. Pelvic Floor Rehabilitation in the Treatment of Fecal Incontinence. Clin Colon Rectal Surg. 2014;27:99-105.

36. Maris A, Devreese AM, D'Hoore A, Penninckx F, Staes F. Treatment options to improve anorectal function following rectal resection: A systematic review. Colorectal Dis. 2013;15:67-78.

37. Visser WS, Te Riele WW, Boerma D, Van Ramshorst B, Van Westreenen HL. Pelvic floor rehabilitation to improve functional outcome after a low anterior resection:
A systematic review. Ann Coloproctol. 2014;30:109-14.

38. Laforest A, Bretagnol F, Mouazan AS, Maggiori L, Ferron M, Panis Y. Functional disorders after rectal cancer resection: Does a rehabilitation programme improve anal continence and quality of life? Colorectal Dis. 2012;14:1231-7.

39. Faaborg PM, Christense P, Buntzen S, Laurberg S, Krogh K. Anorectal function after long-term transanal colonic irrigation. Colorectal Dis. 2010;12:314-9.

40. Rosen H, Robert-Yap J, Tentschert G, Lechner M, Roche B. Transanal irrigation improves quality of life in patients with low anterior resection syndrome. Colorectal Dis 2011;13:e3358. http://www.ncbi.nlm.nih.gov/ pubmed/21689359.

41. Altomare D, Picciariello A, Digennaro R, Ribas Y, De Fazio M. Short-term outcomes of percutaneous tibial nerve stimulation for low anterior resection syndrome: results of a pilot study. Colorectal Dis. 2017; epub.

42. Lundby L, Møller A, Buntzen S, Krogh K, Vang K, Gjedde A, et al. Relief of fecal incontinence by sacral nerve stimulation linked to focal brain activation. Dis Colon Rectum 2011;54:318-23.

43. Ramage L, Qiu S, Kontovounisios C, Tekkis P, Rasheed S, Tan E. A systematic review of sacral nerve stimulation for low anterior resection syndrome. Colorectal Dis. 2015;762-71. http://www.ncbi.nlm. nih.gov/pubmed/25846836.

44. Duelund-Jakobsen J, Lehur PA, Lundby L, Wyart V, Laurberg S, Buntzen S. Sacral nerve stimulation for faecal incontinenceefficacy confirmed from a two-centre prospectively maintained database. Int J Colorectal Dis. 2016;31:421-8.

45. Laurberg S. Sacral nerve stimulation for faecal incontinence: From voodoo to evidence-based medicine. Color Dis. 2011;13:836.

46. Celerier B, Denost Q, Van Geluwe B, Pontallier A, Rullier E. The risk of definitive stoma formation at 10 years after low and ultralow anterior resection for rectal cancer. Colorectal Dis. 2016;18:59-66.

47. Patel UB, Taylor F, Blomqvist L, George C, Evans H, Tekkis P, et al. Magnetic resonance imaging-detected tumor 


\section{ARTíCULO DE REVISIÓN}

response for locally advanced rectal cancer predicts survival outcomes: MERCURY experience. J Clin Oncol. 2011;29:3753-60.

48. Harris DA, Thorne $\mathrm{K}$, Hutchings $\mathrm{H}$, Islam S, Holland G, Hatcher O, et al. Protocol for a multicentre randomised feasibility trial evaluating early Surgery Alone In LOw Rectal cancer (SAILOR). BMJ Open 2016;6:e12496. http:// bmjopen.bmj.com/lookup/doi/10.1136/ bmjopen-2016-012496.

49. Pontallier A, Denost Q, Van Geluwe B, Adam JP, Celerier B, Rullier E. Potential sexual function improvement by using transanal mesorectal approach for laparoscopic low rectal cancer excision. Surg Endosc Other Interv Tech. 2016;30:4924-33.

50. Panteleimonitis S, Ahmed J, Ramachandra M, Farooq M, Harper M, Parvaiz
A. Urogenital function in robotic $v s$ laparoscopic rectal cancer surgery: a comparative study. Int J Colorectal Dis 2017;32:241-8. http://dx.doi.org/10.1007/ s00384-016-2682-7.

51. Kauff DW, Lang H, Kneist W. Risk Factor Analysis for Newly Developed Urogenital Dysfunction after Total Mesorectal Excision and Impact of Pelvic Intraoperative Neuromonitoring-a Prospective 2-Year Follow-Up Study. J Gastrointest Surg. 2017;21:1038-47.

52. Kauff DW, Kronfeld K, Gorbulev S, Wachtlin D, Lang H, Kneist W.

Continuous intraoperative monitoring of pelvic autonomic nerves during TME to prevent urogenital and anorectal dysfunction in rectal cancer patients (NEUROS): a randomized controlled trial. BMC Cancer 2016;16:323. http://bmccancer.biomedcentral.com/ articles/10.1186/s12885-016-2348-4.

53. Larach JT, Bellolio F. Plataformas de acceso transanal en cirugía colorrectal: de la resección local a la resección total del mesorrecto. Rev Chil Cir. 2015;67:214-24.

54. Lorimer PD, Motz BM, Kirks RC, Boselli DM, Walsh KK, Prabhu RS, et al. Pathologic Complete Response Rates After Neoadjuvant Treatment in Rectal Cancer: An Analysis of the National Cancer Database. Ann Surg Oncol. 2017; http://link.springer.com/10.1245/s10434017-5873-8.

55. Kong JC, Guerra GR, Warrier SK, Ramsay RG, Heriot AG. Outcome and Salvage Surgery Following \&quot; Watch and Wait\&quot; for Rectal Cancer after Neoadjuvant Therapy: A Systematic Review. Dis Colon Rectum 2017;60:33545. http://www.ncbi.nlm.nih.gov/ pubmed/28177997. 